\title{
MEMS Inertialsensoren für innovative Applikationen in der Medizintechnik
}

\author{
Dr. Marc Füldner \\ Analog Devices GmbH, Wilhelm-Wagenfeld-Str. 6, 80807 München \\ 089-76903117, marc.fueldner@analog.com
}

\section{Einleitung}

Inertialsensoren wie Beschleunigungssensoren und Drehratensensoren auf Basis der MEMSTechnologie haben sich milliardenfach in Anwendungen der Automobiltechnik (Airbag-Auslösung, Fahrdynamikregelung), im Konsumgüterbereich (Smartphones, Spielkonsolen) und der Industrieautomation (Navigation, Maschinenüberwachung) bewährt. Durch die stetige Verbesserung der Leistungsmerkmale hinsichtlich Genauigkeit, Robustheit, Bauteilgröße, integrierter Funktionen und Leistungsaufnahme ermöglichen MEMS Inertialsensoren zahlreiche neue innovative Anwendungen in der Medizintechnik. Während bisher MEMS Sensoren in der Medizintechnik hauptsächlich unter dem Schlagwort „Bio-MEMS“ als elektrochemische Mikro-Analysesysteme („Lab-On-Chip“) fungieren, liefern MEMS Inertialsensoren präzise Informationen über die Beschleunigung, Geschwindigkeit, Position, Neigung, Vibration, Drehbewegung oder Orientierung von medizinischen Geräten.

Dieser Artikel liefert zunächst einen kurzen Einblick in verschiedene MEMS-Technologien und wie diese für anwendungsoptimierte und leistungsfähige Inertialsensoren eingesetzt werden. Ausgehend vom Funktionsprinzip von MEMS Beschleunigungs- bzw. Drehratensensoren werden anhand von typischen Leistungsanforderungen verschiedener Applikationsbereiche sowohl bereits im Einsatz befindliche Systeme als auch mögliche zukünftige Anwendungen in der Medizintechnik diskutiert.

\section{Anwendungsoptimierte Technologien für MEMS Inertialsensoren}

MEMS Inertialsensoren auf Basis der Silizium-Halbleitertechnik ermöglichen monolithisch integrierte Sensoren. Das heißt, die mechanische MEMS-Struktur zur Bewegungsdetektion und die Signalaufbereitung befinden sich auf einem Silizium-Chip (Abb. 1). Die monolithische Integration liefert die Grundlage für besonders kleine Bauteile und reduzierte Fertigungskosten in der Aufbau- und Verbindungstechnik, da nur ein Silizium-Chip auf ein Substrat montiert und mittels Bonddrähten elektrisch verbunden werden muss. Zudem werden durch die kurze Signalstrecke zwischen der hochimpedanten MEMS-Struktur und der ersten Verstärkerstufe parasitäre Lastkapazitiven minimiert und eine potentielle elektromagnetische Störeinstrahlung verringert. Somit können besonders empfindliche und störunanfällige Sensoren realisiert werden.

Bei erhöhten Anforderungen an die Signalverarbeitung und zusätzlichen Funktionen, wie einer anwendungsspezifischen Programmierung (Messbereich, Filter, Inaktivitäts-/Aktivitätsmonitor, Interrupts etc.), kalibrierten Sensordaten (über Temperatur, Spannungsbereich, Linearität etc.) oder digitalen Schnittstelllen, können hybride Systeme vorteilhaft eingesetzt werden. Bei diesen werden das MEMSElement und der integrierte Schaltkreis jeweils auf separaten Chips gefertigt. Die Trennung der zumeist sehr unterschiedlichen Fertigungstechnologien des MEMS-Elementes und der Schaltkreise erleichtert eine Optimierung hinsichtlich notwendiger Prozessschritte, maximaler Ausbeute und flexibler Fertigungsplanung auf Kosten eines erhöhten Aufwandes in der Aufbau- und Verbindungstechnik. Ein weiterer Vorteil in der hybriden Technologie liegt in der verhältnismäßig einfachen Integration zusätzlicher MEMS-Zellen zur Realisierung von Inertialsensormodulen mit verschiedenen Sensortypen, Sensorachsen und Dynamikbereichen. Derartige „Kombo“-Sensormodule bieten nicht nur mehr Sensorfunktionalität in einem Gehäuse sondern erlauben auch eine höhere Leistungsfähigkeit der Einzelfunktionen, z.B. durch eine gegenseitige Kompensation von Querempfindlichkeiten. 

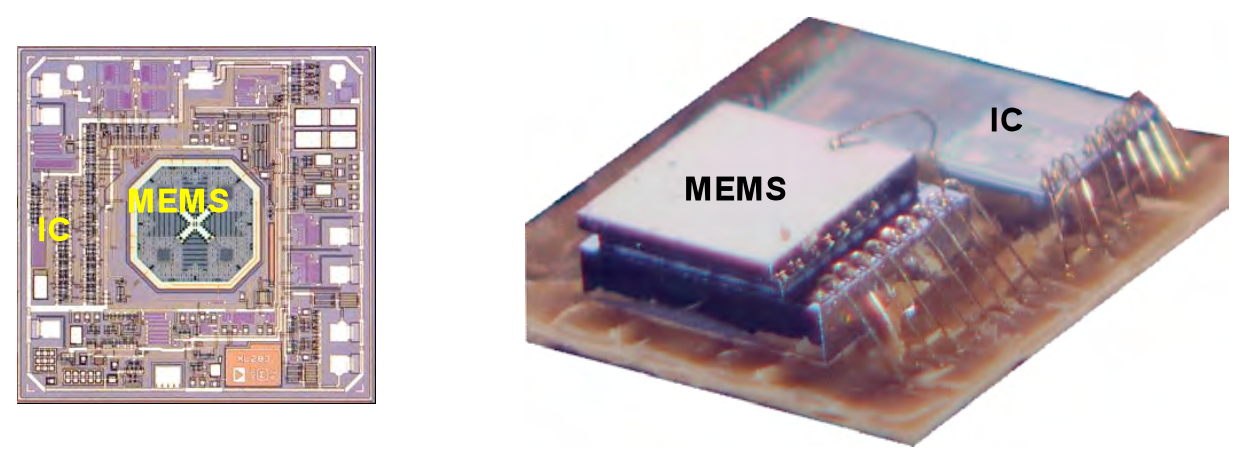

\begin{tabular}{|c|}
\hline Monolithische Integration \\
\hline Hohe Sensor-Empfindlichkeiten \\
\hline Vereinfachte Aufbau- und Verbindungstechnik \\
\hline Geringe Bauteilgrößen
\end{tabular}

Geringe Bauteilgrößen
Hybride Integration

Anwendungsspezifische Funktionen

Verringerte Halbleiter-Fertigungskomplexität

Erhöhte Modularität

Abb. 1: Monolithische und hybride MEMS-Technologie für verschiedene Anforderungsschwerpunkte.

Bei Inertialsensoren beeinflusst die Gehäuse-Technologie im besonderen Maße die Eigenschaften des Sensors. Typische Einflussfaktoren sind Temperaturänderungen und Feuchtigkeitseintrag in das Gehäuse, die über die Leiterplatte und das Gehäuse mechanische Verspannungen in das MEMSElement einprägen können. Die mechanische Verspannung äußert sich dann über ein scheinbares oder verfälschtes Sensorsignal über Temperatur und Zeit.

Abbildung 2 illustriert vereinfacht den Zusammenhang der Sensorgenauigkeit im Sinne von Temperaturdrift, Langzeitdrift über Lebenszeit, Drift durch Montagestress etc. und den Herstellungskosten für verschiedene Gehäuse-Technologien. Keramik-Gehäuse sind aufgrund ihrer hohen Steifigkeit und geringer Feuchtigkeitsaufnahme die erste Wahl für besonders stabile und präzise Sensoren. Darüber hinaus steht in diesem Hohlraumgehäuse der MEMS-Sensor nur über das Substrat mit dem Gehäuse in Verbindung, so dass sich Spannungen kaum bis zu den MEMS-Strukturen entwickeln können. Spritzvergossene Kunststoff-Gehäuse bieten dagegen Vorteile in der Baugröße und den Gehäuse-Kosten. Da die umgebende Spritzmasse das Einkoppeln mechanischer Spannungen aufgrund von Feuchtigkeit oder Temperaturänderungen erleichtert, zeigen derartig verarbeitete Sensoren eine deutlich schlechtere Leistungsfähigkeit bezüglich Sensordrift.

Unabhängig von der Gehäuse-Technologie erlaubt eine integrierte Kalibrierung eine Verbesserung der Sensorgenauigkeit. Dazu wird z.B. jeder einzelne Sensor werksseitig über den spezifizierten Temperatur, Dynamik- oder Versorgungsspannungsbereich gemessen und eine Kompensationskurve im Sensor programmiert. Eine Optimierung des Verhältnisses von Genauigkeit zu Kosten ist hierbei über die Anzahl der Kalibrier-Stützstellen möglich.

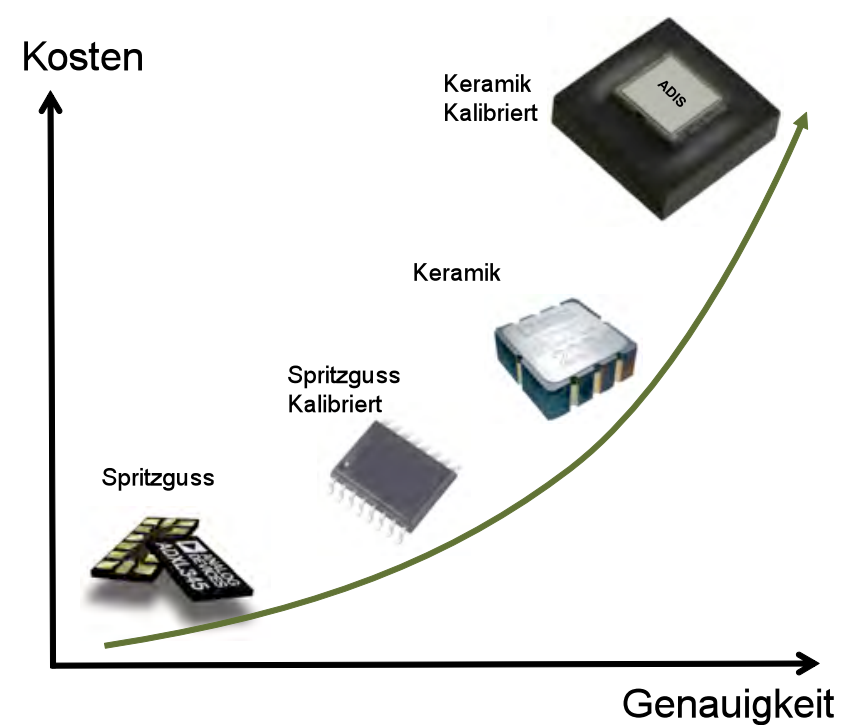

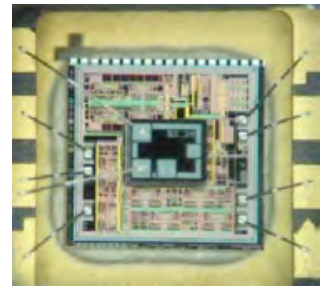

Hohlraumgehäuse

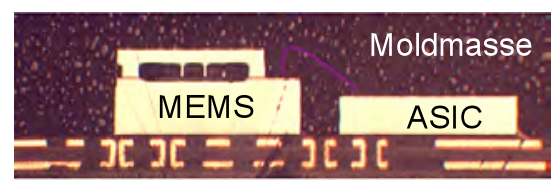

Spritzgussgehäuse

Abb. 2: Vereinfachter Zusammenhang zwischen Sensorgenauigkeit und Herstellungskosten. 
Dieser kurze Überblick über MEMS- und Gehäuse-Technologien verdeutlicht, dass für spezifische Produkt- und Applikationsanforderungen jeweils der Einsatz anwendungsoptimierter Technologien erforderlich ist.

\section{MEMS Beschleunigungssensoren in der Medizintechnik}

Beschleunigungssensoren messen lineare statische oder zeitlich veränderliche Bewegungen über die auftretenden Trägheitskräfte. In diesem Kapitel wird zunächst das Funktionsprinzip eines kapazitiven Beschleunigungssensors dargestellt. Im Vergleich zu piezoresistiven oder piezoelektrischen Sensoren erreichen kapazitive Beschleunigungssensoren hohe Empfindlichkeiten auch bei niedrigen Messfrequenzen und eine hohe Temperaturstabilität. Es wird erläutert, wie spezielle Sensordesigns unterschiedliche Applikationen in der Medizintechnik ermöglichen.

\subsection{Funktionsprinzip eines MEMS Beschleunigungssensors}

Abbildung 3 zeigt den prinzipiellen Aufbau eines kapazitiven MEMS-Beschleunigungssensors nach dem Feder-Masse-Prinzip. Aufgrund der flexiblen Aufhängung (rot) der Inertialmasse (gelb) kommt es bei Auftreten einer Beschleunigung zu einer Relativbewegung zwischen den Messfingern an der Inertialmasse und den Referenzelektroden am Substrat (violett). Diese Relativbewegung bewirkt eine Kapazitätsänderung an der differentiellen Messstruktur, die von der Auswerteelektronik in ein analoges oder digitales Sensorsignal gewandelt wird. Das dargestellte Sensor-Design erlaubt sowohl statische als auch dynamische Beschleunigungsmessungen.

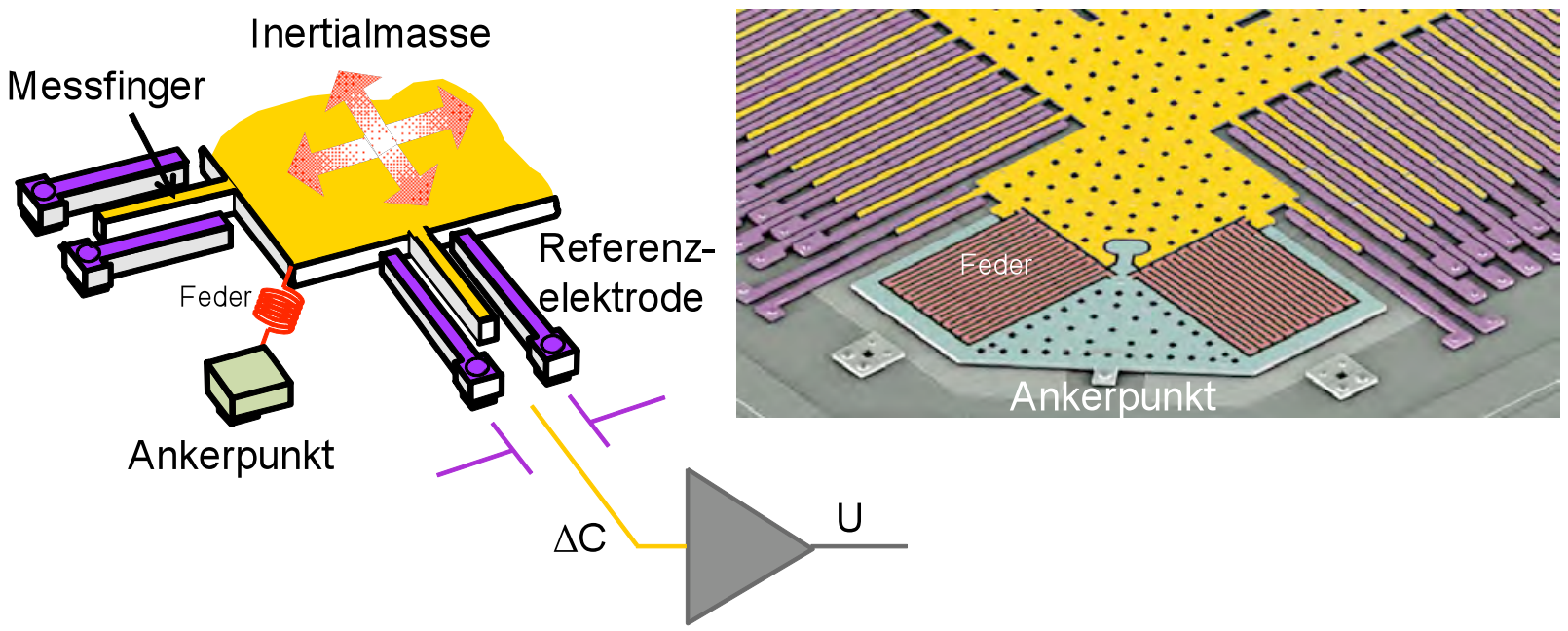

Abb. 3: Prinzip eines kapazitiven Beschleunigungssensors in MEMS-Technologie.

Grundlegende Design-Parameter eines Beschleunigungssensors sind die Federsteifigkeit $k$ der Aufhängung der Inertialmasse und dessen Masse $m$. Die Federsteifigkeit wird beispielsweise durch die Breite, Länge und Anzahl von Mäanderstrukturen definiert. Typische Strukturgrößen liegen im Bereich

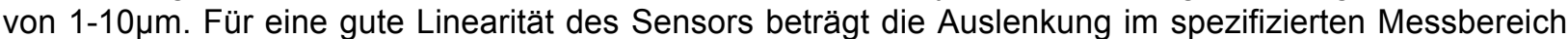
typischerweise nur $1 \%$ des durch den Kondensatorspalt theoretisch möglichen Wertes. Diese Auslenkung im Bereich von 1-10nm entspricht einer Kapazitätsänderung von ca. 1fF und verdeutlicht die hohen Anforderungen an die Signalverarbeitung.

Aus dem Hookeschen Gesetz $m \cdot a=-k \cdot \Delta x$ und der Resonanzgleichung eines Feder-Masse-Schwingers $\omega^{2}=k / m$ erhält man einen einfachen Ausdruck für die Sensor-Empfindlichkeit $S$ :

$$
S=\frac{U}{a} \sim \frac{\Delta C}{a} \sim \frac{\Delta x}{a} \sim \frac{m}{k}=\frac{1}{\omega^{2}}
$$

Das bedeutet, die Resonanzfrequenz $\omega$ bestimmt neben der maximalen Bandbreite $\Delta f_{\max } \sim \omega$ des Sensors auch die Empfindlichkeit und damit wesentlich auch die eingangsbezogene Rauschdichte bzw. das 
Auflösungsvermögen des Sensors. Danach ist die Anforderung einer möglichst hohen Bandbreite und einer möglichst hohen Auflösung prinzipiell gegenläufig und so muss für jede Applikation entschieden werden, welches Sensordesign den besten Kompromiss liefert. Tabelle 1 gibt einige Beispiele für das eingangsbezogene Rauschen bzw. Auflösungsvermögen verschiedener Designs von kapazitiven MEMS Beschleunigungssensoren. Der zur Gruppe der "Low-g“ Beschleunigungssensoren gehörende ADXL203 erzielt durch seine relativ niedrige Resonanzfrequenz von $5.5 \mathrm{kHz}$ eine geringe Rauschdichte $N_{d}$ von nur $0.11 \mathrm{mg} / \sqrt{\mathrm{Hz}} \quad\left(1 \mathrm{mg}=9.81 \mathrm{~m} / \mathrm{s}^{2} \cdot 10^{-3}\right)$. Dagegen ist der "High-g" Sensor ADXL001 mit seiner hohen Resonanzfrequenz von ca. $22 \mathrm{kHz}$ und entsprechend höheren Rauschdichte besonders für Messungen von Beschleunigungen mit hohen Amplituden und Frequenzanteilen geeignet.

In den meisten Fällen liegt die in der Applikation genutzte Bandbreite deutlich unter der Resonanzfrequenz, so dass sich Fertigungsstreuungen der Resonanzfrequenz nicht als Fehler in der Empfindlichkeit auswirken. Zudem wird man durch Tiefpass-Filterung (z.B. RC-Tiefpass oder Mittelwertbildung) die Bandbreite jeweils auf die minimale Anforderung der Applikation begrenzen, da das Sensorrauschen $N$ über die Rauschdichte $N_{D}$ mit der Applikationsbandbreite $\Delta f$ ansteigt:

$$
N=N_{d} \cdot \sqrt{\Delta f}
$$

Am Beispiel des ADXL203 erreicht man mit einer Applikationsbandbreite von $10 \mathrm{~Hz}$ ein Auflösungsvermögen von $0.35 \mathrm{mg}\left(\sim 2.4 \mathrm{~cm} / \mathrm{s}^{2}\right)$ im Vergleich zu $1.1 \mathrm{mg}$ bei $100 \mathrm{~Hz}$.

Der Sensor ADXL345 beinhaltet bereits einen Analog-Digital-Wandler und einen digitalen Tiefpass-Filter. Dies erlaubt in einfacher Weise eine Programmierung der Bandbreite bzw. Datenrate. So kann eine Reduzierung der Bandbreite wie beim analogen Sensor für ein verbessertes Auflösungsvermögen eingesetzt werden, oder aber es kann bei reduzierter Datenrate des Sensors die Stromaufnahme mittels

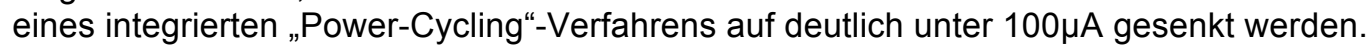

\begin{tabular}{|c|c|c|c|c|c|c|}
\hline Parameter & Einheit & \multicolumn{2}{|c|}{ ADXL203 } & \multicolumn{2}{|c|}{ ADXL345 } & ADXL001 \\
\hline Resonanzfrequenz & $\mathrm{kHz}$ & \multicolumn{2}{|c|}{5.5} & \multicolumn{2}{|c|}{5.5} & 22 \\
\hline $\begin{array}{l}\text { Eingangsbezogene } \\
\text { Rauschdichte } N_{d}\end{array}$ & $\mathrm{mg} / \sqrt{\mathrm{Hz}}$ & \multicolumn{2}{|c|}{0.11} & \multicolumn{2}{|c|}{0.29} & 3.3 \\
\hline $\begin{array}{l}\text { Applikationsbandbreite } \Delta f \\
\text { (Beispiel) }\end{array}$ & $\mathrm{Hz}$ & 10 & 100 & 12.5 & 100 & 10000 \\
\hline $\begin{array}{l}\text { RMS-Rauschen } N \\
\text { (Auflösungsvermögen) }\end{array}$ & $\mathrm{mg}$ & 0.35 & 1.1 & 2.9 & 2.9 & 330 \\
\hline Messbereich & $\mathrm{g}$ & \multicolumn{2}{|c|}{1.7} & \multicolumn{2}{|c|}{$2-16$} & $70-500$ \\
\hline Stromaufnahme & $\mathrm{mA}$ & \multicolumn{2}{|c|}{0.7} & 0.06 & 0.14 & 2.5 \\
\hline Temperaturdrift & $\mathrm{mg} /{ }^{\circ} \mathrm{C}$ & & & \multicolumn{2}{|c|}{0.4} & \\
\hline Gehäuse & & \multicolumn{2}{|c|}{ Keramik } & \multicolumn{2}{|c|}{ Kunststoff } & Keramik \\
\hline Schnittstelle & & \multicolumn{2}{|c|}{ Analog } & \multicolumn{2}{|c|}{ Digital } & Analog \\
\hline
\end{tabular}

Tabelle 1: Beispiele von verschiedenen MEMS-Beschleunigungssensoren zur Einhaltung spezifischer Anforderungen an das Auflösungsvermögen, der Bandbreite oder der Stromaufnahme.

\subsection{Applikationen in der Medizintechnik}

\section{Neigung}

Eine Applikation mit geringen Anforderungen an die Bandbreite (typ. $<10 \mathrm{~Hz})$ und den Messbereich $(<1 \mathrm{~g})$ aber möglichst hoher Auflösung ist die Neigungsmessung. Sogenannte Inklinometer oder Neigungswinkelmesser sind Beschleunigungssensoren, welche die zur Erdnormalen gerichtete Erdbeschleunigung $1 \mathrm{~g} \sim 9.81 \mathrm{~m} / \mathrm{s}^{2}$ messen. Der vektorielle Anteil der Erdbeschleunigung, der auf die jeweilige Sensorachse wirkt (Messgröße $a_{x}, a_{y}, a_{z}$ ), ist ein direktes Maß für die Neigung $\Theta$ des Sensors relativ zum Horizont (Abb. 4). 

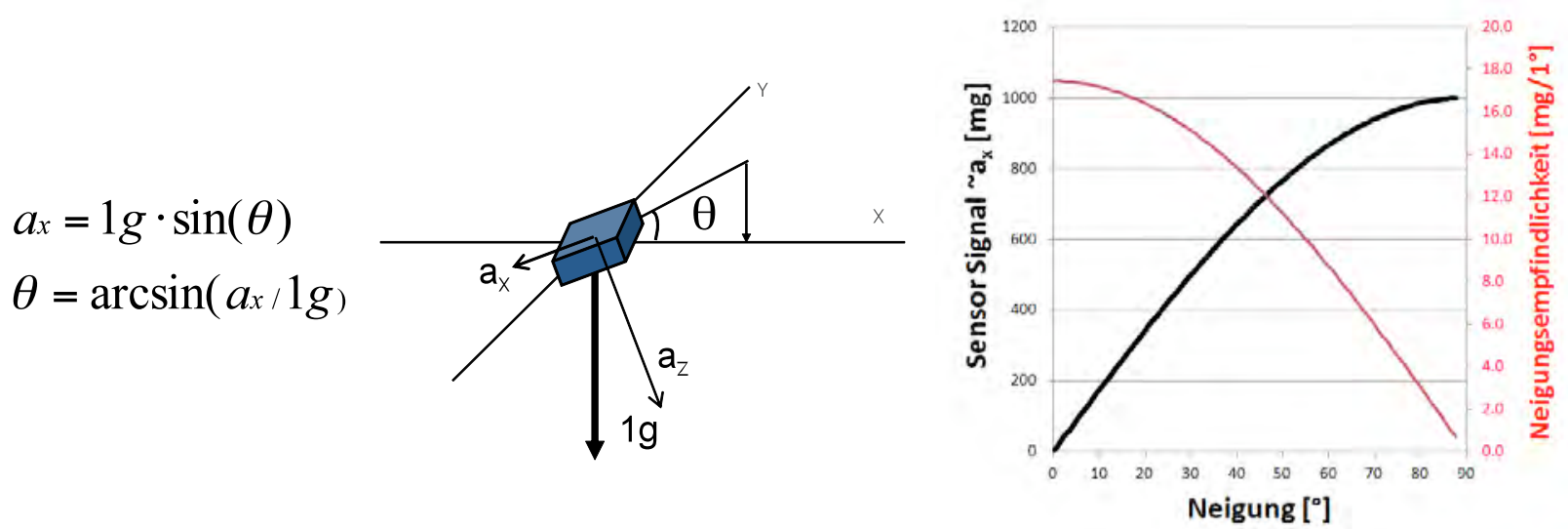

Abb. 4: Prinzip der Neigungsmessung mit einem Beschleunigungssensor.

Bei relativ kleinen Neigungen muss der Sensor für eine Winkelauflösung von $1^{\circ}(\sim 0.017 \mathrm{rad})$ ein Auflösungsvermögen von mindestens $1 \mathrm{~g}^{*} \sin (0.017 \mathrm{rad})=17 \mathrm{mg}$ besitzen. Aufgrund der trigonometrischen Beziehung zwischen dem Neigungswinkel und der Erdbeschleunigung steigt die Anforderung an die Auflösung mit dem Neigungswinkel. Bei einer Neigung von z.B. $70^{\circ}$ beträgt der Sensor-Signalhub einer $1^{\circ}$-Winkeländerung nur noch $6 \mathrm{mg}$. Im Extremfall einer $90^{\circ}$ Neigung ist die Neigungsempfindlichkeit, also die Sensorsignaländerung pro Neigungsänderung, gleich null (vergleiche Abbildung 4, rechte Grafik). Die vollständige $360^{\circ}$ Lageerkennung im Raum erfolgt daher unter Verwendung mehrachsiger Beschleunigungssensoren [1].

Ein Applikationsbeispiel aus der Medizintechnik, bei der die Neigungsinformation eines MEMS Beschleunigungssensors eingesetzt werden kann, liegt im Bereich der Aspirationsprophylaxe. Aspiration bezeichnet das Eindringen von Festkörpern oder Flüssigkeiten wie z.B. Nahrung oder Magensäure in die Atemwege. Verbleiben die Fremdkörper in den Atemwegen oder der Lunge, etwa aufgrund unzureichender Schutzreflexe, kann dies zur Erstickung oder schwerwiegenden Entzündungen führen. Überdurchschnittlich gefährdete Personen sind bettlägerige Patienten mit einer flachen Liegeposition. Daher ist eine Maßnahme der Aspirationsprophylaxe die aufrechte Liegeposition während und direkt nach der Nahrungsaufnahme. Die Überwachung der aufrechten Liegeposition übernimmt dabei der am Patienten oder Bett platzierte Beschleunigungssensor (Abb. 5, links). Neben einer einfachen Warnfunktion bei einem zu flachen Liegewinkel sind auch steuernde Maßnahmen wie eine automatische Änderung der Bettposition denkbar.

Weitere mögliche Einsatzgebiete für Neigungssensoren bieten bildgebende Geräte zur Computertomographie oder Sonographie, bei denen die Abbildungsqualität von der Positionierungsgenauigkeit und Ausrichtung des Senders und der Bildsensoren abhängt. So wird beispielsweise in der Computertomographie das entsprechende Körperteil zunächst aus verschiedenen Richtungen geröntgt. Die zu jeder Einzelaufnahme gehörende Richtungsinformation geht dann als wesentlicher Faktor in die rechnerbasierte Rekonstruktion der eigentlichen Abbildung ein. Bei Geräten mit gesundheitsgefährdenden Strahlenquellen kommt der korrekten Ausrichtungsüberwachung zur Vermeidung von Fehlbestrahlungen zudem eine Sicherheitsfunktion zu. Auch in mobilen Geräten zur Vermessung von Gelenkwinkeln oder dem Wirbelsäulenverlauf (Goniometer) können MEMS Neigungssensoren aufgrund ihrer kleinen Baugröße und geringer Leistungsaufnahme vorteilhaft eingesetzt werden.

Neben der erwähnten hohen Auflösung ist für Neigungsanwendungen eine möglichst geringe NullpunktDrift notwendig, da diese direkt als Neigung interpretiert werden würde. Ein geeigneter Baustein für derartige Anwendungen ist der ADXL203 im Keramikgehäuse (Tabelle 1) oder dessen digitale und kalibrierte Varianten der ADIS162xx Serie. 

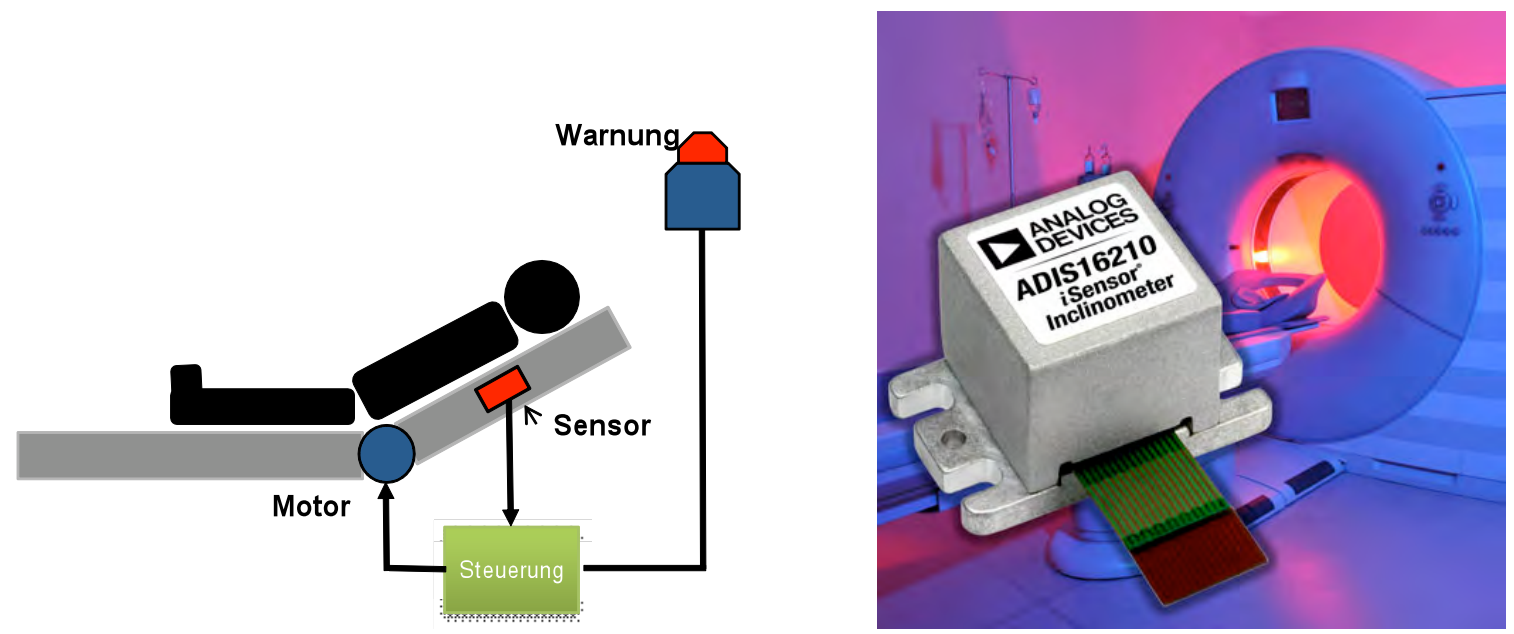

Abb. 5: Neigungsapplikationen in der Medizintechnik: Bestimmung der Liegeposition zur Aspirationsprophylaxe (links), Ausrichtung der Strahlungsquelle bei medizinischen Scannern (rechts)

\section{Bewegung}

Ein Beispiel für eine Applikation mit mittleren Bandbreiten $(<100 \mathrm{~Hz})$ und Messbereichen $(<10 \mathrm{~g})$ ist die Aktivitätsüberwachung und Bewegungsanalyse von Patienten oder älteren Personen in der Altenpflege. Prinzipiell erlaubt die Beschleunigungsmessung a über Integration über die Zeit $t$ eine Berechnung der zeitlichen Entwicklung der Geschwindigkeit $v$ und Position $x$ :

$$
\begin{aligned}
& v(t)=v\left(t_{0}\right)+\int_{t_{0}}^{t} a(t) \cdot d t \\
& x(t)=x\left(t_{0}\right)+\int_{t_{0}}^{t} v(t) \cdot d t \sim \frac{1}{2} \cdot a \cdot t^{2}
\end{aligned}
$$

Die Startposition $x\left(t_{0}\right)$ bzw. die Anfangsgeschwindigkeit $v\left(t_{0}\right)$ ist entweder bekannt, für die Applikation nicht relevant oder wird mit Hilfe anderer Messmethoden (z.B. GPS) ermittelt.

Durch die Integration summieren sich Nullpunktfehler des Sensors allerdings schnell zu großen Fehlern in der Positionsbestimmung auf. Nullpunktfehler sind z.B. durch das Sensor-Eigenrauschen, Temperaturänderungen oder auch durch die Applikation bedingt. Beispielsweise entspricht eine geringe Neigung von $1^{\circ}$ einer Beschleunigung von ca. $17 \mathrm{mg}$. Unter dieser Randbedingung würde nach $t=1 \mathrm{~min}$ die berechnete Wegstrecke $300 \mathrm{~m}$ ergeben, obwohl der Körper tatsächlich ruht und nur leicht geneigt ist. Die Beschleunigung aufgrund einer Neigung kann nicht von einer tatsächlichen Bewegung unterschieden werden. Theoretisch ließe sich die z-Achse zur Differenzierung verwenden. Das Signal dieser Achse geht allerdings für kleine Neigungen im Sensorrauschen verloren, da die Neigungsempfindlichkeit für die zAchse (=Neigungsausrichtung nahe $90^{\circ}$ ) praktisch null ist (vergleiche Abbildung 4, rechtes Diagramm). Navigationslösungen nutzen daher fast immer auch Drehratensensoren zur unabhängigen Messung des lokalen Koordinatensystems, um eine g-Kompensation durchzuführen [2].

Dennoch erlaubt die zeitliche Analyse der Bewegungsprofile eines Beschleunigungssensors wertvolle Rückschlüsse über die Aktivität der überwachten Personen, wie z.B. Phasen des Gehens, Laufens oder Liegens (Abb. 6). Für ein System mit z-Achsen Beschleunigungssensor ist eine ruhende aufrechte Position (Sitzen, Stehen) durch ein nahezu konstantes Signal von $1 \mathrm{~g}$ charakterisiert. Durch Gehen bzw. Laufen wird diesem 1g-Signal ein Wechselsignal im Takt der Schrittfolge mit unterscheidbarer Amplitude und Frequenz aufmoduliert. Beim Liegen ist die Beschleunigung in Richtung der z-Achse des Beschleunigungssensors nahezu null.

Angefangen von einfachen Alarmfunktionen, etwa wenn eine Liegeposition außerhalb typischer Ruhezeiten auf eine akute Notsituation hinweisen könnte, könnten derartige Systeme frühzeitig Bewegungsmuster erkennen, um etwa einen Herzschrittmacher an die jeweilige Belastungssituation anzupassen, Schlafstörungen zu diagnostizieren, Rehabilitationsaktivitäten zu überwachen oder die Behandlung von Krankheiten mit Bewegungsstörungen (z.B. das Zittern von Parkinson-Patienten) zu unterstützen. 

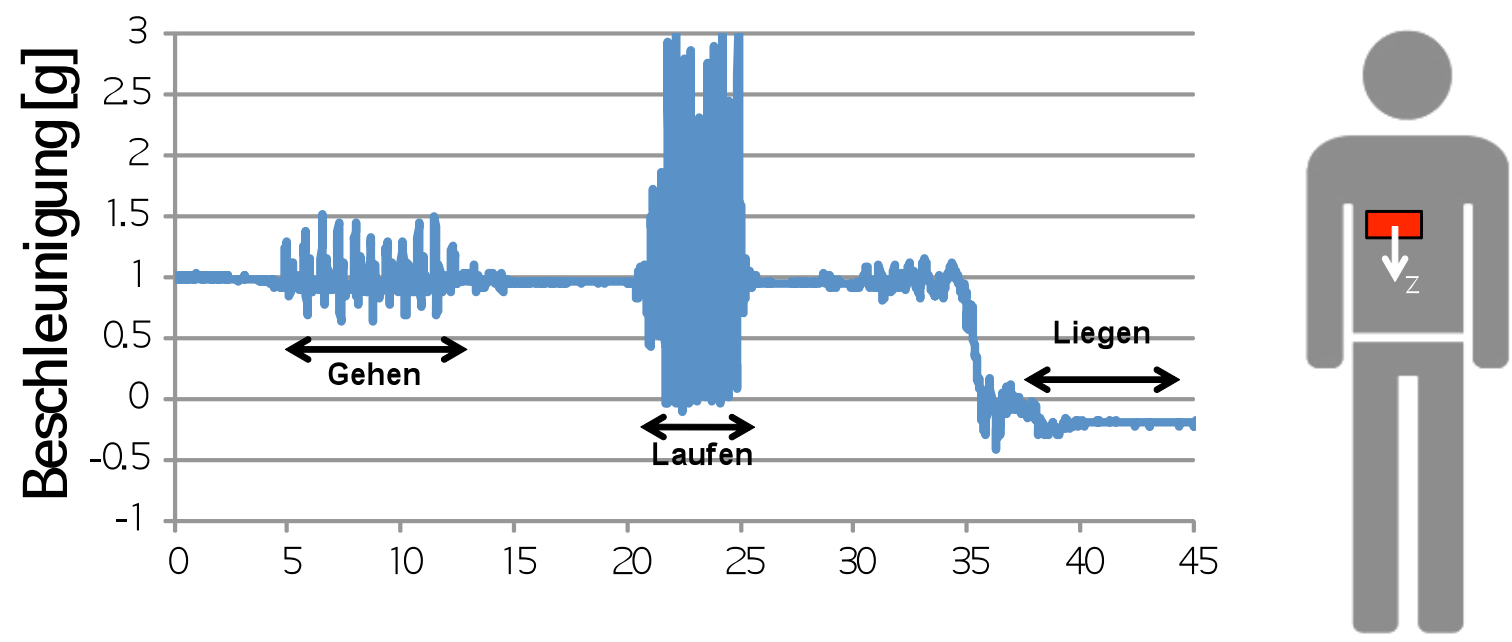

Abb. 6: Beispielhafte Bewegungsanalyse unterschiedlicher Aktivitäten mit einem mobilen System mit zAchsen Beschleunigungssensor.

Ein weiteres Anwendungsbeispiel im Bereich der dynamischen Bewegungsmessung ist ein mobiles Ersthelfergerät für kardiopulmonale Reanimationen. Die Durchführung einer Herzdruckmassage zur Reanimation erfolgt häufig nicht den medizinischen Empfehlungen entsprechend, da viele Ersthelfer vor Ort mit dieser Notfallmaßnahme nicht vertraut sind. In diesen Fällen kann ein mobiles Gerät den Ersthelfer unterstützen, indem ein integrierter Beschleunigungssensor die Frequenz und Drucktiefe der Massage in Echtzeit misst (Abb. 7). Bei Abweichungen zur empfohlenen Frequenz (ca. 100 Drücke pro Minute) und Kompressionstiefe (ca. 3,5-5cm) wird dies dem Ersthelfer audiovisuell signalisiert, so dass die Massage angepasst werden kann.

Die wichtigsten Sensor-Anforderungen der genannten mobilen Applikationen zur Bewegungsanalyse sind ein niedriges Rauschen zur präzisen Beschleunigungsmessung, eine geringe Leistungsaufnahme für den Langzeiteinsatz des batteriebetriebenen Geräts und eine kleine Baugröße. Diese Anforderungen werden durch leistungsfähige MEMS Beschleunigungssensoren wie dem digitalen Sensor ADXL345 (Tabelle 1) oder dessen analoge Varianten ADXL32x/33x erfüllt.
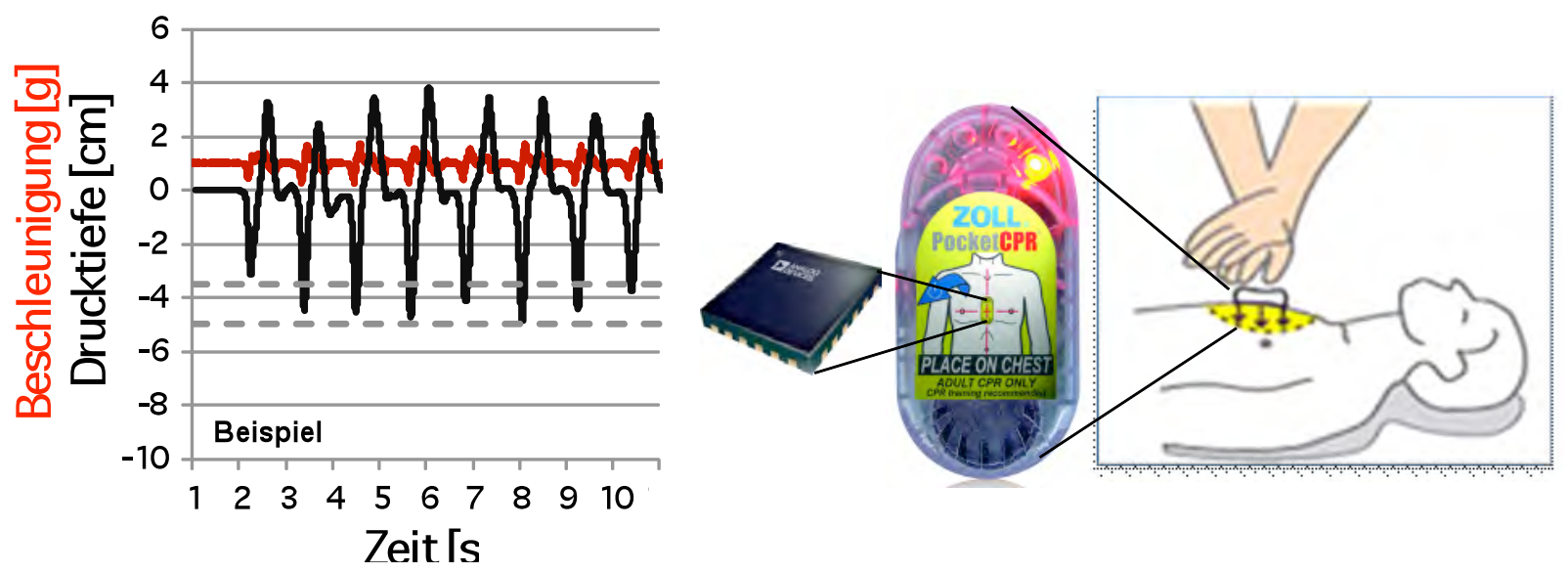

Abb. 7: Ersthelfergerät mit Beschleunigungssensor zur Messung der Drucktiefe bei einer Herzmassage [3].

\section{Vibration/Schock}

Hohe Bandbreiten der Beschleunigungsmessung bis zu $20 \mathrm{kHz}$ und hohe Messbereiche bis zu $100 \mathrm{~g}$ werden in Applikationen zur Geräteüberwachung gefordert. Bei den häufig hochwertigen medizinischen Geräten kann eine Überwachung und Analyse des typischen Vibrationsspektrums bewegter Baugruppen, 
wie z.B. Motoren, Generatoren, Lager, Lüfter oder Pumpen frühzeitig eine Schädigung melden, um Instandhaltungskosten und Ausfallzeiten zu reduzieren. Schleichende Schädigungen derartiger Baugruppen äußern sich häufig in einer Änderung der Vibrationsamplituden oder der Ausbildung höherharmonischer Störfrequenzen durch nichtlineare Bewegungsanteile. Abbildung 8 (linke Darstellung) zeigt ein exemplarisches Vibrationsprofil mit einer Grundfrequenz bei ca. $1.3 \mathrm{kHz}$ und dessen Harmonische. Durch eine Programmierung von Frequenzbändern mit Amplitudenschwellwerten, wie sie z.B. der Baustein ADIS16228 bereits integriert anbietet, können anwendungsspezifische Vorwarnstufen für die Instandhaltung oder ein kritischer Alarm zur Notabschaltung definiert werden. Der hohe Messbereich bis zu 100g ergibt sich aus der Tatsache, dass die Beschleunigung a einer Vibration mit dem Quadrat der Vibrationsfrequenz $f$ ansteigt:

$$
\begin{aligned}
& x(t)=A \cdot \sin (2 \pi f \cdot t) \\
& a(t)=\frac{d^{2} x(t)}{d t^{2}}=-(2 \pi f)^{2} \cdot x(t)
\end{aligned}
$$

So treten bei einer harmonischen Schwingung $x(t)$ mit der Amplitude $A=1 \mu \mathrm{m}$ bei einer Frequenz $f=100 \mathrm{~Hz}$ Beschleunigungen bis zu $0.4 \mathrm{~g}$ auf. Bei einer Frequenz von $1 \mathrm{kHz}$ sind es bei gleicher Amplitude bereits ca. $40 \mathrm{~g}$. Auch bei Anwendungen zur Detektion von mechanischen Schocks, die auf ein Gerät eingewirkt haben, ist eine hohe Bandbreite zur korrekten Amplitudenmessung notwendig. Wird ein Sensor mit zu geringer Bandbreite eingesetzt, kann es zu einer Verfälschung der Messung kommen (Abb. 8, rechts). Derartige Anwendungen dienen z.B. der Klärung von Gewährleistungsansprüchen bei unsachgemäßer Lieferung oder bei einem Sturz im Betrieb. Ein „Schock“-Sensorsystem könnte eine eventuell notwendige Neukalibrierung empfindlicher Präzisionsgeräte nach dem Einwirken hoher Beschleunigungen signalisieren. Ein Baustein, der sowohl die Anforderung einer hohen Bandbreite als auch eines hohen Messbereiches erfüllt, ist beispielsweise der analoge Sensor ADXL001 (Tabelle 1) oder eine digitale oder mehrachsige Variante aus der ADIS1622x Serie.

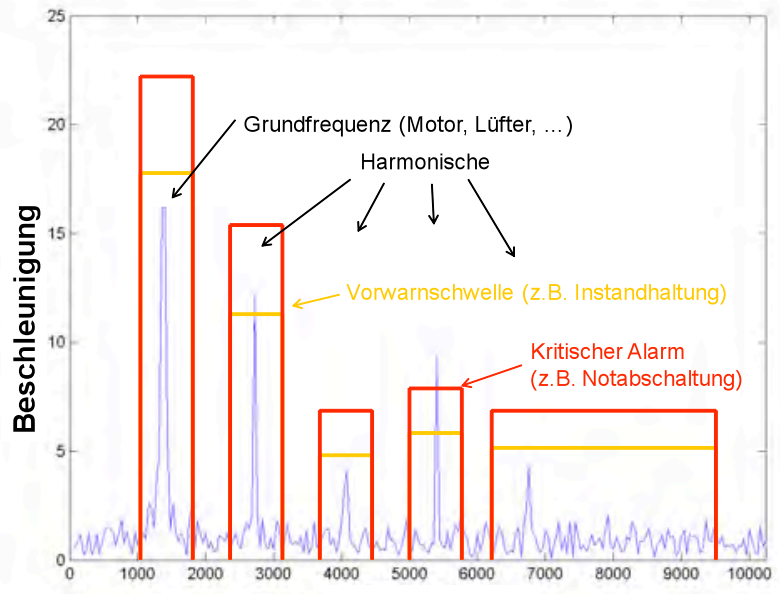

Frequenz $[\mathrm{Hz}]$

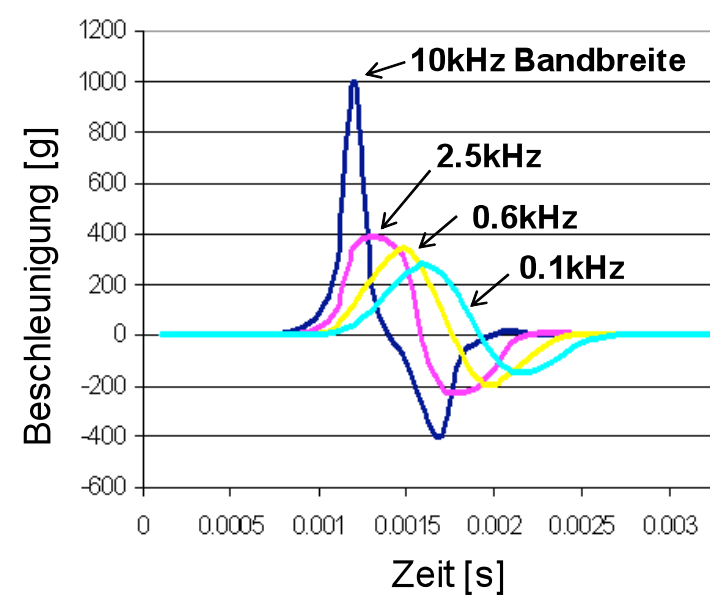

Abb. 8: Monitoring des Vibrationsprofils medizinischer Geräte zur Minimierung von Instandhaltungskosten und Ausfallzeiten (links). Unverfälschte Detektion eines mechanischen Schocks durch breitbandige Beschleunigungssensoren (rechts).

\section{MEMS Drehratensensoren in der Medizintechnik}

In Abgrenzung zu einem Beschleunigungssensor gibt ein Drehratensensor (Gyroskop) die Winkelgeschwindigkeit oder Drehrate (Einheit [rad/s] oder [\%]) eines Körpers an. In vielen Applikationen ist nicht die Drehrate die eigentliche Messgröße sondern die über die zeitliche Integration bestimmte aktuelle Richtung und Orientierung des Körpers. Prinzipiell kann eine Drehrate auch mit Hilfe eines Beschleunigungssensors unter dem Einfluss der Zentrifugalkraft gemessen werden. Allerdings hängt die Zentrifugalkraft vom Abstand zum Rotationszentrum ab und ist daher im Grenzfall einer Rotation um den Schwerpunkt des Körpers gleich null. Ein Drehratensensor nutzt dagegen die positionsunabhängige Corioliskraft und erreicht eine etwa 100fach größere Empfindlichkeit. In diesem Kapitel wird zunächst 
kurz das Funktionsprinzip und Design dieser Sensoren diskutiert, um dann Anwendungsbeispiele aus der Medizintechnik vorzustellen.

\subsection{Funktionsprinzip eines MEMS Drehratensensors}

Ein Drehratensensor ist vereinfacht dargestellt ein Beschleunigungssensor mit einer oszillierende Masse, bei dem unter der Wirkung der Corioliskraft eine Drehrate in ein Beschleunigungssignal transformiert wird. Die Corioliskraft $F_{c}$ ist eine Trägheitskraft, die auf bewegte Massen $m$ der Geschwindigkeit $v$ in einem rotierenden System (Winkelgeschwindigkeit $\omega$ ) wirkt:

$\stackrel{\prime}{F}_{c}=m \cdot \stackrel{r}{a_{c}}=2 \cdot m \cdot(\stackrel{r}{v} \times \stackrel{r}{\omega})$

Für eine oszillierende Masse mit Frequenz $f$ und Amplitude $A$ ist $v=2 \pi f A$, so dass die Winkelgeschwindigkeit gegeben ist durch:

$\omega=\frac{a_{c}}{4 \cdot \pi \cdot f \cdot A}$

Die Gleichung beschreibt die wesentlichen Komponenten eines Sensorelements in einem Drehratensensor (vergleiche Abbildung 9):

1) Eine Inertialmasse wird mit Hilfe eines gesteuerten elektro-mechanischen Kammantriebs in eine Schwingung der Frequenz $f$ und Amplitude $A$ versetzt (Nenner der Gleichung).

2) Ein Rahmen entkoppelt die senkrecht zur Schwingungsrichtung wirkende Coriolisbeschleunigung $a_{c}$ und misst diese mit kapazitiven Messfingern wie ein Beschleunigungsensor (Zähler der Gleichung).

Mit typischen Parametern der MEMS-Technologie lassen sich Drehraten bis zu $0.005^{\circ} / \mathrm{s}$ auflösen. Dies entspricht anschaulich der Bewegung des Stundenzeigers einer Uhr.
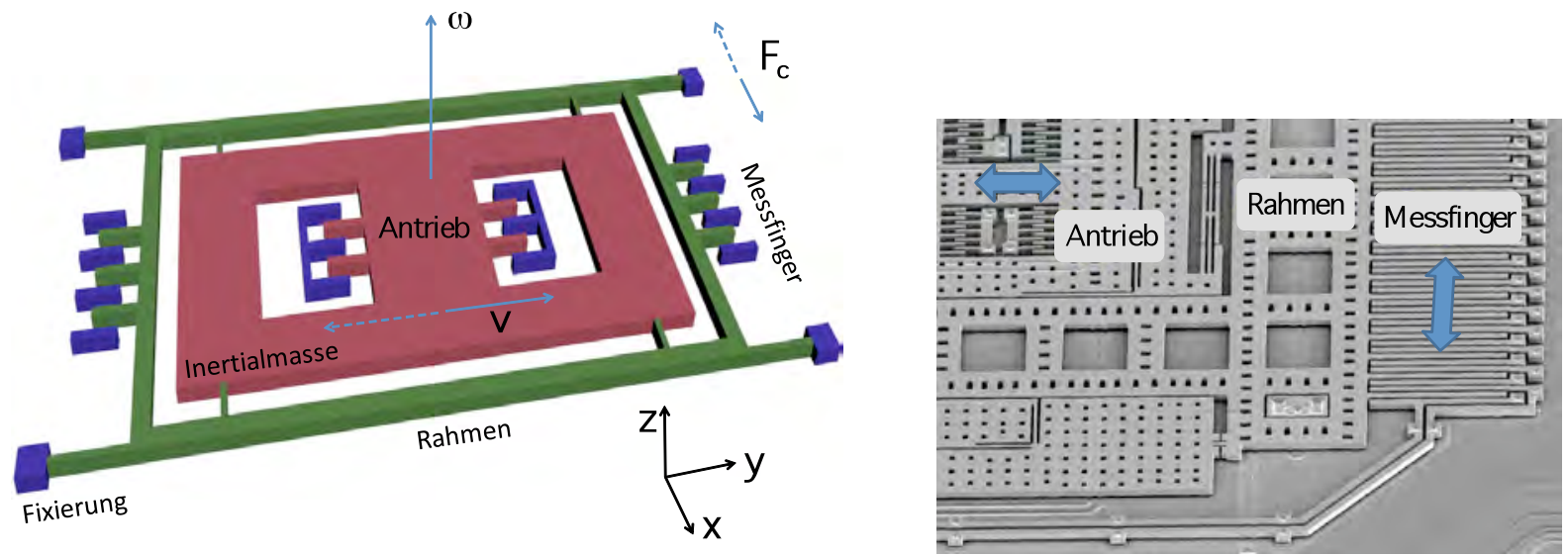

Abbildung 9: Schematischer Aufbau eines Drehratensensors und Realisierung in MEMS-Technologie

In der schematischen Darstellung der Abbildung 9 ist ein Drehratensensor in seiner einfachsten Form durch ein einzelnes Sensorelement dargestellt. Es ist allerdings leicht ersichtlich, dass ein einzelnes Sensorelement nicht nur auf eine Drehrate sondern auch sehr empfindlich auf lineare Beschleunigungen reagiert. So führt beispielsweise eine Beschleunigung in x-Richtung direkt zu einer Bewegung des Rahmens und somit zu einem Messsignal, das von einem vermeintlichen Drehratensignal nicht zu unterscheiden ist. Selbst in Applikationen, in denen man zunächst keine störenden externen Beschleunigungen vermuten würde, liegt praktisch immer ein Querempfindlichkeitsfehler aufgrund der $1 \mathrm{~g}$ Erdbeschleunigung vor [4]. Auch Auslenkungen der Sensorstruktur aufgrund von Drifteffekten oder thermomechanischen Verspannungen bewirken direkt ein virtuelles Drehratensignal.

Eine deutliche Verbesserung der Robustheit wird durch die Verwendung differentieller Sensor-Designs erreicht (Abbildung 10). Durch einen Differenzverstärker werden die um $180^{\circ}$ phasenverschobenen Drehratensignale verstärkt. Im Gegensatz dazu eliminieren sich die durch Beschleunigungen oder 
Drifteffekte erzeugten gleichphasigen Signale (Gleichtaktfehler). Kleine Unterschiede im Amplituden- und Phasengang einer einfach-differentiellen Anordnung würden allerdings zu einer unvollständigen Auslöschung und somit zu einer verbleibenden Querempfindlichkeit führen. Daher werden in der „QuadSensor"-Technologie in der aktuellen Generation von Drehratensensoren von Analog Devices vier Sensor-Elemente in Gestalt zweier Differenzverstärker derart verschaltet, dass sich die Einzelsignale $S_{x}$ aufgrund der Corioliskraft bzw. Drehrate addieren. Gleichtaktstörsignale werden dagegen unterdrückt. Durch die zusätzliche Differenz-Bildung löschen sich geringe Asymmetrien zwischen den beiden zweifachen Elementen weiter aus, was sich in einer weiteren Erhöhung der Störfestigkeit bemerkbar macht.

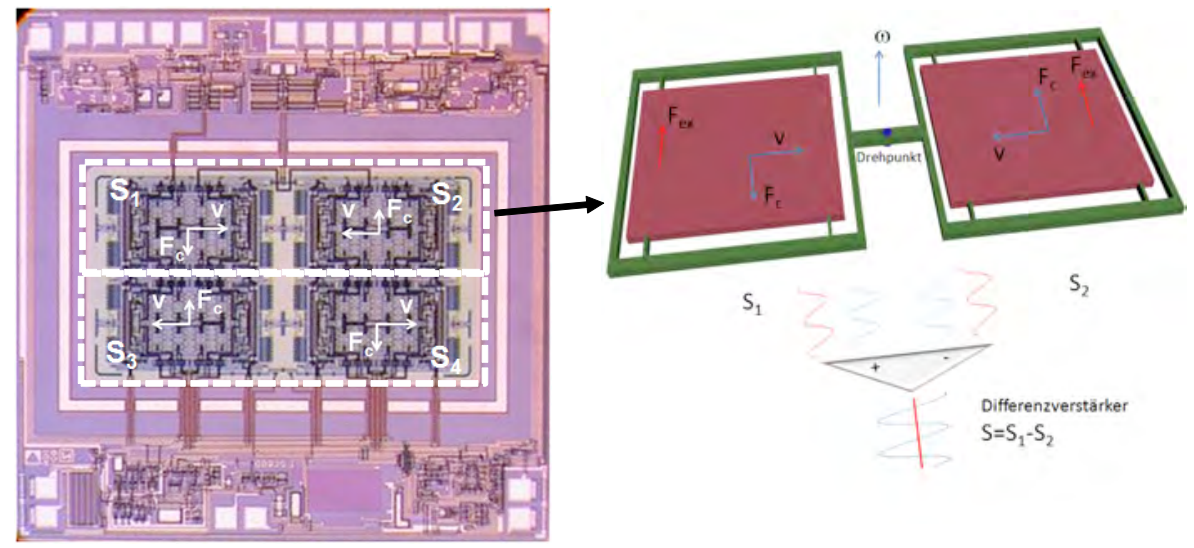

Drehraten Signal:

$S(\omega)=\left(S_{1}-S_{2}\right)-\left(S_{3}-S_{4}\right) \approx 4 \cdot S_{x}(\omega)$

Störsignal (Beschleunigung, Drift):

$S(a)=\left(S_{1}-S_{2}\right)-\left(S_{3}-S_{4}\right) \approx \Delta_{1}-\Delta_{2} \approx 0$

Abbildung 10: Drehratensensor in zweifach-differentieller "Quad-Sensor"-Technologie mit konstruktiver Addition von Drehraten-Signalen bei gleichzeitiger Unterdrückung von Gleichtaktfehlern wie Linearbeschleunigungen oder Nullpunktdrift.

\subsection{Applikationen in der Medizintechnik}

In den meisten Applikationen wird das Signal $S$ [rad/s] eines Drehratensensors zur Bestimmung der zeitlichen Änderung der Bewegungsrichtung $\theta$ [rad] über die Zeit integriert:

$$
\theta(t)=\theta\left(t_{0}\right)+\int_{t_{0}}^{t} S(t) \cdot d t
$$

Aus der Richtungsinformation eines Drehratensensors und der Geschwindigkeitsinformation eines Beschleunigungssensors (vergleiche Abschnitt 3.2) pro Zeitintervall kann die räumliche Bewegung berechnet werden.

Da ein Drehratensensor nur die zeitliche Entwicklung einer Richtung oder Winkels angeben kann, muss der initiale absolute Winkel $\theta\left(t_{0}\right)$ alternativ bestimmt werden. Dieser wird z.B. zum Zeitpunkt $t_{0}$ durch eine bekannte Startposition definiert oder durch Sensoren mit bekannter Richtungsreferenz gemessen (z.B. Magnetfeldsensor im Erdmagnetfeld, Beschleunigungssensor im Erdschwerefeld, optische oder elektromagnetische Marker). Für gewöhnlich wird der Abgleich mit einer Referenz periodisch wiederholt, um die integrationsbedingten Driftfehler zu minimieren.

Ein Drehratensensor kann unterstützend in den in Abschnitt 3.2 besprochenen NeigungssensorApplikationen eingesetzt werden. Dies gilt insbesondere für dynamische Systeme wie z.B. den medizinischen Scannern. Eine hohe Dynamik erzwingt ein erhöhtes Eigenrauschen des Beschleunigungssensors und damit Messfehler aufgrund der notwendigen Sensorbandbreite. Außerdem können im dynamischen System auftretende Vibrationen das eigentliche Messsignal der Neigung 
überlagern bzw. verfälschen. In diesen Fällen kann die zusätzliche (relative) Winkelinformation des Drehratensensors für ein insgesamt robusteres und präziseres Neigungssensorsystem genutzt werden.

Ein weiteres Einsatzgebiet sind Navigationssysteme für die computerunterstützte Chirurgie. Die Überlagerung der aktuellen Position der chirurgischen Instrumente mit einer Abbildung des Operationsobjektes (z.B. eine CT-, Röntgen- oder Ultraschall-Aufnahme) ermöglicht besonders präzise und minimal invasive Eingriffe. Die heutzutage standardmäßig eingesetzte optische Verfolgung von Instrumenten mit einer Stereokamera und optischen Markern hat den Nachteil, dass bei fehlender Sichtverbindung keine Positionsbestimmung möglich ist, und die Marker für eine hinreichende Erkennung relativ groß sein müssen. Alternative elektromagnetische Trackingsysteme bieten in dieser Hinsicht Vorteile, sind aber empfindlich gegenüber Feldstörungen und daher ausfallanfällig. Vielversprechend sind daher auch hier Systeme, die verschiedene Ansätze zur Positionsbestimmung kombinieren und so die jeweiligen Schwächen der Trackingmethoden ausgleichen können. Dazu liefert ein in das Operationsinstrument integrierter Drehraten- und Beschleunigungssensor bzw. ein integriertes Inertialsensormodul (IMU) zusätzliche Daten zur Modellierung der räumlichen Bewegung (Abb. 11). Die Fusion der unterschiedlichen Sensordaten zu einer insgesamt präziseren und robusteren Systemantwort gelingt mit einer applikationsspezifischen Kalman-Filterung, die mit der notwendigen Datenverarbeitung (1g Kompensation bei der Geschwindigkeits- und Positionsbestimmung, Integralbildung etc.) in einer zentralen Recheneinheit erfolgt. Neben der bereits diskutierten hohen Stabilität gegen verschiedene äußere Einflüsse bieten MEMS Inertialsensoren eine relativ kleine Baugröße, ein geringes Gewicht und eine niedrige Stromaufnahme für derartige Anwendungen.

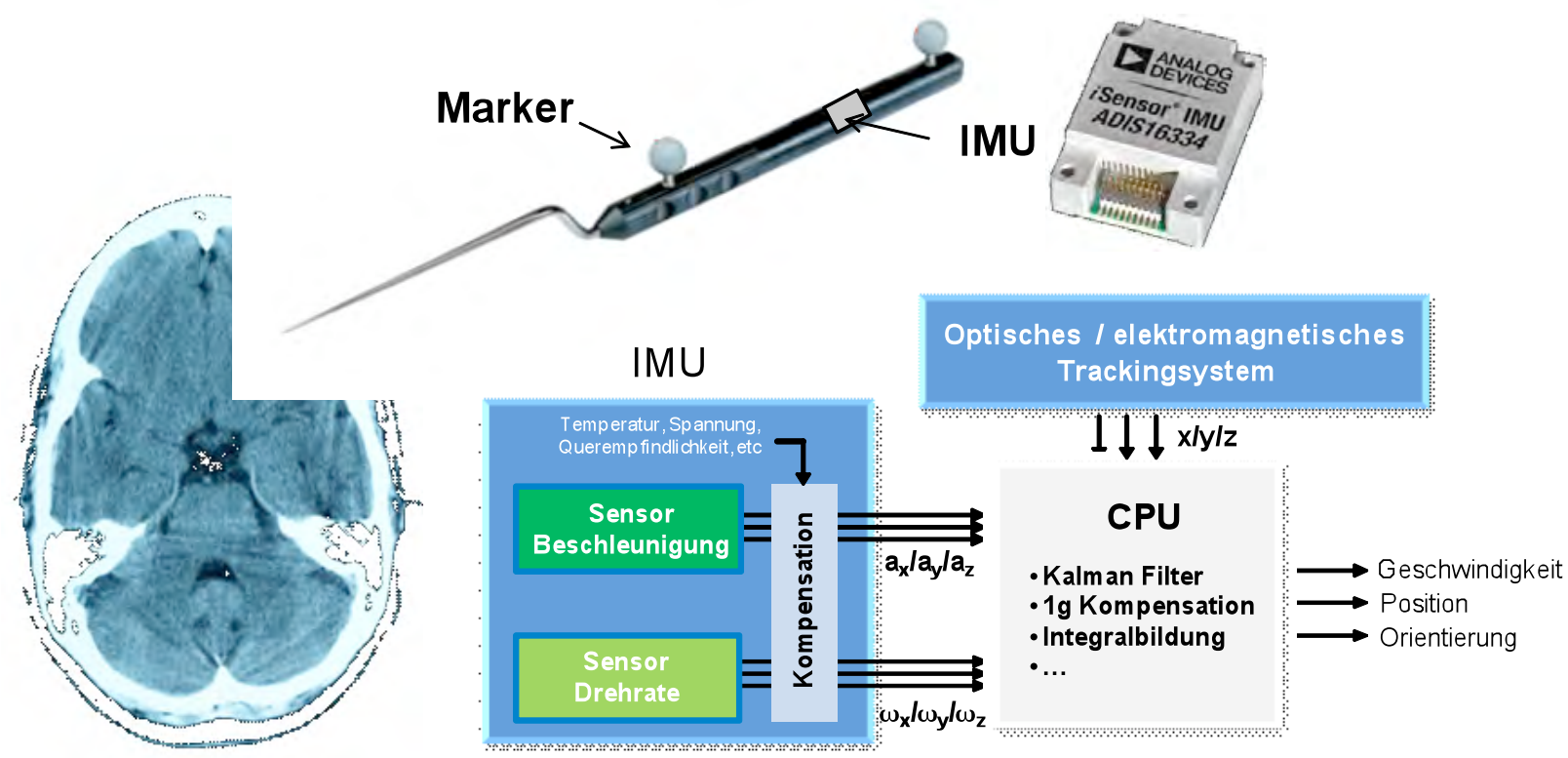

Abbildung 11: Prinzip eines chirurgisches Navigationssystems mit einem MEMS Inertialsensormodul (IMU) zur Erhöhung der Präzision und Zuverlässigkeit.

Einen Schritt weiter gehen Applikationen in der Prothetik, bei denen MEMS Inertialsensoren nicht nur bei der Navigation der Operationsinstrumente helfen, sondern die optimale Positionierung und Ausrichtung der Prothesen durch eine individuelle Vermessung des erkrankenden Bewegungsapparates bestimmen. Bei dem in Abbildung 12 dargestellten System handelt es sich um ein Navigationsgerät mit einem Inertialsensorsystem zur Implantation eines künstlichen Kniegelenks. Während das Knie des Patienten einem kompletten Bewegungsablauf unterzogen wird, berechnet das System auf Basis der aufgenommenen Positionen und der Dynamik die genauen Schnittwinkel zum Durchtrennen der Knochen. Die Kniegelenkprothese ist mit ca. 100.000 Operationen jährlich in Deutschland eine der häufigsten Prothesen. Derzeit weisen schätzungsweise 30 Prozent aller herkömmlichen, rein mechanischen Operationen eine nicht komplett zufriedenstellende Ausrichtung der Implantate auf. 


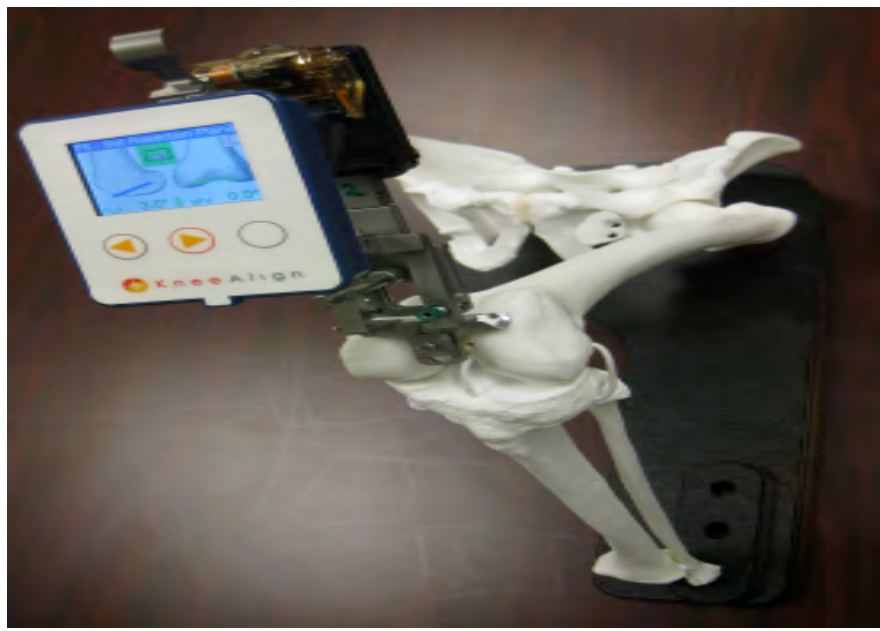

Abbildung 12: Navigationssystem mit MEMS Intertialsensoren in der Kniegelenkprothetik [5]

\section{Fazit}

MEMS Inertialsensoren erreichen durch den Einsatz anwendungsoptimierter Technologien und SensorDesigns eine Leistungsfähigkeit, die neben den bewährten Konsumgütern und Produkten der Automobiltechnik neue Applikationen in der Medizintechnik ermöglichen. MEMS Inertialsensoren zeichnen sich durch ihre geringe Bauteilgröße, geringe Stromaufnahme, hohe Genauigkeit und Robustheit bei verhältnismäßig niedrigen Kosten aus. Tabelle 2 fasst die in diesem Artikel diskutierten Applikationsbereiche und einige Produktbeispiele zusammen. Mit der zunehmenden Verbreitung von MEMS Intertialsensoren und der kontinuierlichen Weiterentwicklung ihrer Leitungsfähigkeit ist in Zukunft mit vielen weiteren innovativen Applikationen in der Medizintechnik zu rechnen.

\begin{tabular}{|l|l|c|c|c|}
\hline \multicolumn{3}{|c|}{ Beschleunigungssensor } & \multicolumn{2}{c|}{ Drehratensensor / IMU } \\
\hline Neigung (absolut) & \multicolumn{1}{|c|}{ Bewegung } & Vibration/Schock & Neigung (relativ) & Navigation \\
\hline Liege-/Bettposition & $\begin{array}{l}\text { Patienten- } \\
\text { überwachung }\end{array}$ & Geräteüberwachung & bildgebende Geräte & $\begin{array}{c}\text { chirurgische } \\
\text { Navigation }\end{array}$ \\
\hline bildgebende Geräte & Aktivitätsanalyse & Gerätegewährleistung & & Prothetik \\
\hline Goniometer & Reanimation & & & \\
\hline
\end{tabular}

Tabelle 2: Beispiele für Medizintechnik Applikationen mit MEMS Inertialsensoren

\section{Quellenverzeichnis}

[1] Analog Devices, Applikationsschrift AN-1057

[2] An introduction to inertial navigation, O. J. Woodman, Technical Report 696, University of Cambridge

[3] Customer Case Study, www.analog.com/en/content/customer-case-studies/cs_Zoll_Medicals_PalmSized_CPR_Dev_Customer_Story/fca.html

[4] H. Weinberg, Gyro Mechanical Performance: The Most Important Parameter, Analog Devices, Technical Article, MS-2158

[5] Customer Case Study, www.analog.com/en/mems-sensors/mems-inertial-measurementunits/products/customer-case-studies/cs_orthalign/resources/fca.html 